\title{
Prime DHCP: A Prime Numbering Address Allocation Mechanism for MANETs
}

\author{
Yuan-Ying Hsu and Chien-Chao Tseng
}

\begin{abstract}
Most address allocation schemes rely on broadcasting for address solicitation and/or duplicate address detection. In this paper, we propose a Prime DHCP scheme that can allocate addresses to the hosts of a MANET without broadcasting over the whole MANET. Prime DHCP makes each host a DHCP proxy of the MANET and run a prime numbering address allocation algorithm individually to compute unique addresses for address allocation. The concept of DHCP proxies and the prime numbering address allocation algorithm together eliminate the needs for broadcasting in the MANET. Performance results show that Prime DHCP can significantly reduce the signal overhead and the latency for hosts to acquire addresses.
\end{abstract}

Index Terms-MANET, address allocation, prime, multi-hop.

\section{INTRODUCTION}

A mobile ad hoc network (MANET) offers high flexibility in building communication networks without the needs of base stations. However, the lack of infrastructure in a MANET also causes problems in applying legacy network protocols. In this paper, we focus on the problem of address allocation in MANETs.

Traditionally, a user can either configure the address of a host manually or have the host acquire its address dynamically through certain dynamic methods, such as Dynamic Host Configuration Protocol (DHCP) [1]. However, manual address configuration is very troublesome or even inapplicable to MANETs, and dynamic configurations normally involve broadcasting that is very expensive in MANETs and may cause the broadcast storm problem [2].

In this paper, we propose a Prime $D H C P$ scheme for address allocation without broadcasting in the whole MANET during the address allocation process. In the proposed prime DHCP, each host serves as a DHCP proxy that can assign addresses to new hosts by running a proposed Prime Numbering Address Allocation (PNAA) algorithm individually to compute unique addresses for address allocation. The use of DHCP proxies and the PNAA together eliminate the need for broadcasting in the whole MANET.

\section{RELATED WORKS}

Several dynamic address allocation schemes have previously been proposed for MANETs. According to the prior research [5], they can be classified into three categories:

Manuscript received January 23, 2005. The associate editor coordinating the review of this letter and approving it for publication was Prof. CarlaFabiana Chiasserini. This work is sponsored by National Science Council under grant numbers NSC 93-2219-E-009-001 and NSC 94-2213-E-009-005.

Y.-Y. Hsu and C.-C. Tseng are with the Department of Computer Science and Information Engineering, National Chiao Tung University, Hsinchu, Taiwan, R.O.C. (e-mail: \{yyhsu, cctseng\}@csie.nctu.edu.tw).

Digital Object Identifier 10.1109/LCOMM.2005.08032.
1) Best effort allocation: Schemes in this category can not guarantee address uniqueness. Prophet scheme [8] proposes a complex address generation function for each host to generate a sequence of addresses to be assigned to new coming neighbors. Furthermore, even with a large address space, prophet scheme may still needs some mechanisms, such as Duplicate Address Detection (DAD) or weakDAD [4], to resolve address conflicts. DAD will cause broadcast storm problem and weakDAD will introduce extra packet overhead by adding MAC address into IP headers for all data packets.

2) Centralized allocation: A centralized server is deployed to manage all addresses. DHCP [1] is a typical example, but it needs broadcasting for both server discovery and DAD; the former could be eliminated by a special arrangement [3]. ODACP [5] attempts to reduce broadcasting by having the server broadcast advertisement periodically, so a new host can directly register its addresses to the server. A longer advertisement interval does help to reduce the overhead of broadcasting, but it also results in longer latencies for hosts to obtain addresses.

3) Decentralized allocation: A host could acquire an address by itself or from a neighbor and then performs DAD to ensure the uniqueness of the address. In AAA [6], hosts randomly select an address in the range of 169.254/16. In MANETconf [7], each host stores all addresses used in the MANET, and a new coming host acquires an address from one of its neighbors. The neighbor then broadcasts a query, on behalf of the new host, for DAD throughout the network. Mohsin [10] employs a buddy system for address allocation, but it is difficult to manage address blocks among all MANET hosts.

\section{PRIME DHCP MECHANISM}

As mentioned in the previous section, almost all previous address allocation schemes for MANETs rely on broadcasting for server discovery or DAD. We propose Prime DHCP to achieve these two functionalities without broadcasting. Prime DHCP configures each host as a DHCP proxy, so all hosts are eligible to assign addresses and a new host can acquire an address simply from its neighbors. Besides, each DHCP proxy runs PNAA individually to compute unique addresses for address allocation so that DAD is not required in prime DHCP.

\section{A. Prime Numbering Address Allocation Algorithm}

In order to eliminate the necessity of DAD, we propose a PNAA algorithm to guarantee the uniqueness of addresses. PNAA is originated from the canonical factorization theorem of positive integers, that is, every positive integer can be written as a product of prime numbers in a unique way. 


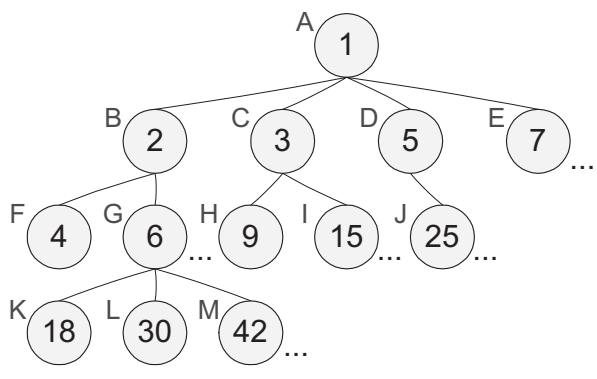

Fig. 1. An example of address allocation tree.

Figure 1 gives an example of addresses a DHCP proxy can assign. The root proxy of a MANET has an address of 1, and can allocate prime numbers, in ascending order, to new hosts attached to it. For a non-root DHCP proxy, it can assign the address equal to its own address multiplied by the unused prime number, starting from the largest prime factor of its own address. Take the host $\mathrm{G}$ with address 6 for example; the largest prime factor of 6 is 3 and the sequence of addresses host $\mathrm{G}$ can assign is, $6 * 3,6 * 5,6 * 7$, and so on up to the largest address bounded by the address space. Each node needs to maintain its allocation status to record the last assigned address.

With PNAA, no two proxies can generate the same address, and thus DAD is not necessary during the process of address resolution. Besides, each proxy can easily derive, from its own address, the address of its parent proxy.

\section{B. Operations of Prime DHCP}

We use Figure 2 as an example MANET to illustrate the operations of Prime DHCP. It should be noted that Figure 1 is just a logical tree of the address allocation. Once a host obtains an address, it can move freely and continuously use the address as long as it is still in this network. Figure 2 is a possible physical topology of MANET hosts in Figure 1. Without loss of generality, we assume that the address space of this MANET is 128 in the following discussion. Furthermore, the messages can be forwarded by any proactive routing protocols for MANETs.

When a new mobile host $N$ joins a MANET, it issues a DHCP_Discover broadcast message as a normal DHCP client does. When the neighbor hosts, $L, B$, and $F$, receive the message, they start serving as DHCP proxies of the host $N$. Rather than forwarding the broadcast message further, each DHCP proxy runs PNAA algorithm to generate an address, and then encapsulates the address in a DHCP_Offer message. Note that proxy $L$ relays the $D H C P$ Discover to its parent proxy $G$ for help because its minimum assignable address is 150, which exceeds the address space 128 . Therefore, host $N$ receives three DHCP_Offer messages offering addresses 66, 10 , and 8 , respectively, from DHCP proxies $G, B$, and $F$. Host $N$ chooses the smallest address 8 to prevent the tree from growing too fast, and broadcasts its choice in a DHCP_Request. Again, its neighbors do not flood the message further, but proxy $L$ relays the message to its parent proxy $G$. Finally, the chosen proxy $F$ updates its address allocation status and

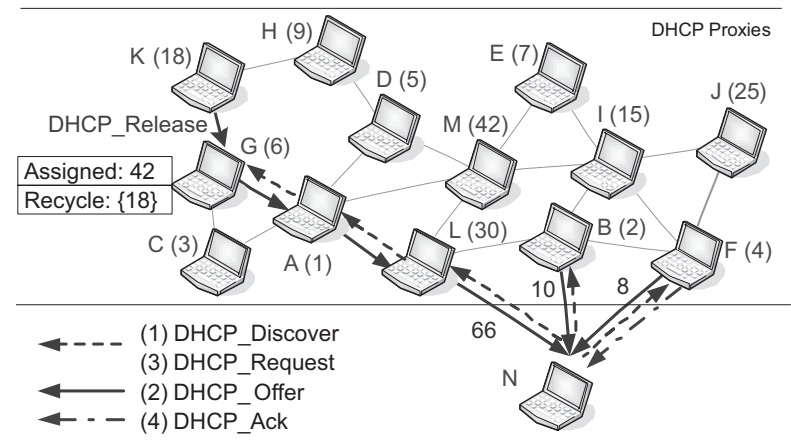

Fig. 2. An Example of MANET topology with a new coming host.

then sends a $D H C P \_A c k$ to host $N$ for confirmation. Consequently, instead of whole-MANET broadcasts, DHCP_Offer and DHCP_Request incur just a single-hop broadcast, which is necessary anyway in the wireless environment, and possibly seldom multi-hop unicasts.

In order to avoid the address leak problem, a host should depart gracefully by informing its parent of leaving, and each host maintains a recycle list to record the allocation statuses for its departed children in its allocation status. For example, proxy $K$ in Figure 2 is leaving, so it sends a DHCP_Release message to its parent proxy $G$. When proxy $G$ receives a DHCP_Discover from a new node, it will first give out the lowest recycled address and inform the new node the allocation status associated with the offered address. If the root is about to leave, it informs its greatest descendent to be the new root proxy. However, it is likely that a host may leave gracelessly, packets may be lost, or MANETs may merge or split. In the next subsection, we will explain how the prime DHCP handles these exceptions.

\section{Exception Handling of Prime DHCP}

Most message losses, except DHCP_Release that can be treated as a graceless departure, can be recovered by having the sender set a timer and resend the messages when the timer expires. For a host left gracelessly, its address may become not usable. In order to reclaim leaked addresses, the root proxy periodically broadcasts a $D H C P \_$Recycle message to ask all hosts report their address allocation statuses, including addresses they have given out or recycled. By gathering statuses from existing hosts, the root proxy can reconfigure the address allocation tree, and inform each proxy of updated allocation status. The DHCP_Recycle broadcast message could be piggybacked in the messages of MANET routing protocol, DSDV [9] for example, so no additional overhead will be introduced by the address recycling.

Network merging and partition can also be detected by periodically recycle process. When two MANETs merge, a root can receive allocation statuses from nodes in the other network, detect address conflicts, and ask one of the two conflicting nodes to inquire a new address. On the other hand, although network partition will not result in address conflicts, the split MANET needs a new root for address recycle. If a node misses several recycle messages, it may claim to be the new root by sending a DHCP_Recycle after a backoff 
TABLE I

QUALITATIVE ANALYSIS OF ADDRESS ALLOCATION MECHANISMS

\begin{tabular}{cccccc}
\hline & DHCP & MANETconf & ODACP & Prophet & PrimeDHCP \\
\hline Uniqueness & Yes & Yes & Yes & No & Yes \\
Signal overhead & $O(4 l)$ & $O(2 l)$ & $O(2 l)$ & $O(n / 2)$ & $O(n / 2)$ \\
Latency & $O(4 t d)$ & $O(2 t d)$ & $O(2 t d)$ & $O(2 t)$ & $O(2 t)$ \\
Complexity & Low & High & Low & High & Low \\
\hline
\end{tabular}

time reversely proportional to its address. This can make the node with the largest address the new root to increase address utilization.

\section{PERformance Evaluation}

Table 1 shows the qualitative analysis of the proposed prime DHCP and other address allocation mechanisms. Assume the numbers of hosts and links are $n$ and $l$, respectively; the network diameter is $d$; and the average one-hop latency is $t$. First, Prophet is the only method that can not guarantee the uniqueness of addresses. Second, DHCP needs to perform server discovery and DAD, so at least $4 l$ hosts need to process signal packets and the latency is $4 * t * d$. MANETconf and ODACP need to perform DAD and server advertisement respectively, and thus the overhead and latency are $2 l$ and $2 * t * d$. Prophet and Prime DHCP both send requests to neighbors only, so the overhead is the average degree $(n / 2)$ of each node in the network and the latency is $2 t$, assuming that the address space is sufficient. At last, MANETconf and Prophet scheme involve more complicated computation in address allocation.

Since the behaviors of address assignment in Prime DHCP and Prophet scheme are similar, the latency and overhead of Prime DHCP can be referred to [8]. Here we focus the analysis on how recycle period affects the address utilization, i.e., the percentage of the effective addresses that Prime DHCP has given out or can possibly assign currently. Suppose the arrival and departure of hosts follow a Poisson distribution with the mean rates $\lambda$ and $\mu$, respectively. The address utilization $U_{k}$ before the $k t h$ recycle can be represented as Equation (1), where $N$ is the size of address space; $\tilde{n}\left(C_{k}\right)$ is the number of hosts in the MANET at the time of $k t h$ recycle; and $\bar{N}_{\text {subtree }}$ is the average number of descendants of a host.

$U_{k}=1-\frac{\tilde{n}\left(C_{k}\right) * \mu * \text { graceless percentage } * \bar{N}_{\text {subtree }}}{N}$.

Figure 3 shows the address utilization $U_{k}$ at different time instances for different recycle periods $(c)$, where $N=256$, $\lambda=0.8$ and $\mu=0.9$. As shown in the figure, when the recycle period becomes shorter, the recycle process will be performed more frequently but utilization will be better. We can also observe from Figure 4 that although the utilization decreases as the recycle period increases, the speed of descent is slow if the address space is sufficiently large. Therefore, address recycling introduces only slight overhead if the MANET has an address space greater than class B, and this could be achieved by configuring the MANET as a private network.

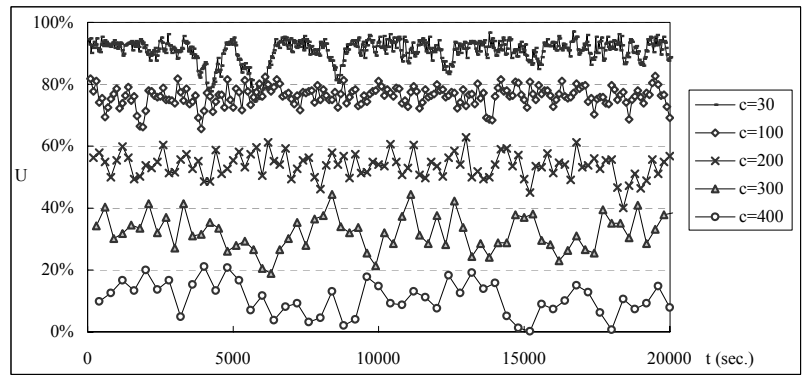

Fig. 3. The effect of recycle period on the address utilization.

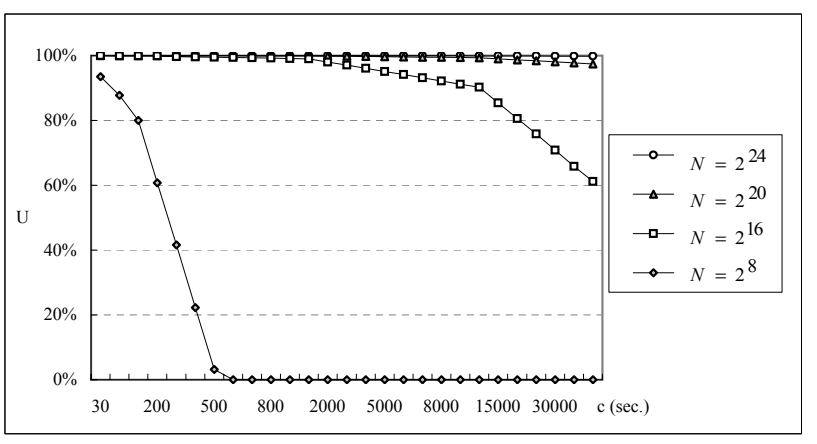

Fig. 4. The effect of address space on the address utilization.

\section{CONCLUSION}

We have proposed a Prime DHCP for address allocation in MANETs. The proposed Prime DHCP is simple but very effective in terms of signal overhead and address allocation latency. We make each MANET host a DHCP proxy and thus DHCP signaling messages need not flood all over the whole MANET. In addition, each proxy assigns addresses according to the prime number tree, so DAD broadcasting is also unnecessary. In the future, we plan to adopt Prime DHCP to improve the efficiency of MANET routing.

\section{REFERENCES}

[1] R. Droms, "Dynamic host configuration protocol," RFC 2131, Mar. 1997.

[2] S.-Y. Ni, Y.-C. Tseng, Y.-S. Chen, and J.-P. Sheu, "The broadcast storm problem in a mobile ad hoc network," in Proc. ACM/IEEE International Conference on Mobile Computing and Networking, pp. 151-162, 1999.

[3] Y.-Y. Hsu, Y.-C. Tseng, C.-C. Tseng, C.-F. Huang, J.-H. Fan, and H.-L. $\mathrm{Wu}$, "Design and implementation of two-tier mobile ad hoc networks with seamless roaming and load-balancing routing capability," IEEE QShine 2004.

[4] N. H. Vaidya, "Weak duplicate address detection in mobile ad hoc networks," in ACM International Symposium on Mobile Ad Hoc Networking and Computing (MobiHoc'02), pp. 206-216, June 2002.

[5] Y. Sun and E. M. Belding-Royer, "A study of dynamic addressing techniques in mobile ad hoc networks," Wireless Communications and Mobile Computing, April 2004.

[6] C. E. Perkins, J. T. Malinen, R. Wakikawa, E. M. Belding-Royer, and Y. Sun, "IP address autoconfiguration for ad hoc networks," draft-ietfmanetautoconf-01.txt, IETF MANET Working Group, July 2000.

[7] S. Nesargi and R. Prakash, in Proc. MANETconf: Configuration of Hosts in a Mobile Ad Hoc Network, INFOCOM 2002, pp. 1059-1068.

[8] H. Zhou, L. M. Ni, and M. W. Mutka, "Prophet address allocation for large scale MANETs," in Proc. INFOCOM 2003, pp. 1304-1311.

[9] C. E. Perkins and P. Bhagwat, "Highly dynamic Destination Sequenced Distance Vector (DSDV) routing for mobile computers," in Proc. ACM SIGCOMM '94 Conf. Communications Architectures, Protocols and Applications, ACM Press, pp. 234-244, 1994.

[10] M. Mohsin and R. Prakash, "IP address assignment in mobile ad hoc networks," in Proc. IEEE MILCOM, pp. 856-861, Sept. 2002. 